JOURNAL OF ALGEBRAIC STATISTICS

Issue in honor of S. E. Fienberg

Volume 10, No. 1, 2019, p.128-136

https://jalgstat.library.iit.edu/

ISSN $\quad 1309-3452$

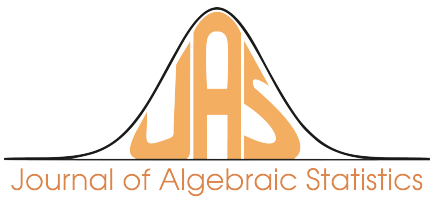

\title{
Strongly Robust Toric Ideals in Codimension 2
}

\author{
Seth Sullivant ${ }^{1}$ \\ ${ }^{1}$ Department of Mathematics, North Carolina State University, Raleigh, NC 27695
}

\begin{abstract}
A homogeneous ideal is robust if its universal Gröbner basis is also a minimal generating set. For toric ideals, one has the stronger definition: A toric ideal is strongly robust if its Graver basis equals the set of indispensable binomials. We characterize the codimension 2 strongly robust toric ideals by their Gale diagrams. This gives a positive answer to a question of Petrović, Thoma, and Vladoiu in the case of codimension 2 toric ideals.
\end{abstract}

2000 Mathematics Subject Classifications: 13P10, 14M25

Key Words and Phrases: toric ideal, Graver basis, robust, Hilbert basis

\section{Introduction}

A homogeneous ideal is robust if its universal Gröbner basis is also a minimal generating set. Although one typically expects the universal Gröbner basis to be much larger than a minimal generating set (and hence most ideals are far from robust), there are a surprising number of examples of ideals that are robust. Usually these examples have rich underlying combinatorics. Three well-known examples are: the ideals of maximal minors of generic matrices of indeterminates $[2,9]$, the vanishing ideal of the closure of an affine linear space in $\left(\mathbb{P}^{1}\right)^{n}[1]$, and toric ideals of Lawrence type (see [8, Chapter 7$]$ ).

Let $A \in \mathbb{Z}^{d \times n}$ be an integer matrix of rank $d$, and $\mathbb{K}[p]:=\mathbb{K}\left[p_{1}, \ldots, p_{n}\right]$ the polynomial ring in $n$ indeterminates. The toric ideal associated to the matrix $A$ is the binomial ideal

$$
I_{A}=\left\langle p^{u}-p^{v}: u, v \in \mathbb{N}^{n}, A u=A v\right\rangle
$$

Properties of the generating set of $I_{A}$ and the geometry of the corresponding variety are determined by combinatorial properties of the matrix $A$, and many conditions can be expressed in terms of linear algebra over the integers. Boocher and Robeva [4] initiated a systematic study of robustness of toric ideals and introduced the word "robust". They showed that a set of quadratic binomials generate a robust ideal if and only if it is the direct sum of ideals of maximal minors of $2 \times n_{i}$ generic matrices on disjoint sets of variables. Since these ideals are toric ideals of Lawrence type, one wonders if all robust toric ideals must be

Email addresses: smsulli2@ncsu.edu (S. Sullivant) 
of Lawrence type. Petrović, Thoma, and Vladoiu [7] studied this problem by introducing an oriented matroid concept they call "bouquets", which we explain below. They also introduced a strengthening of robust for toric ideals, which they called $\emptyset$-Lawrence, and we call strongly robust, that involves looking at a superset of the universal Gröbner basis called the Graver basis (explained in Section 2).

One motivation for studying strongly robust toric ideals comes from algebraic statistics. Recall that the generating set of a toric ideal is called a Markov basis. This is because the binomial generators can be used as a set of moves to perform a random walk on the fiber $\mathcal{F}(u)=\left\{v \in \mathbb{N}^{n}: A u=A v\right\}$ (see [5]). While any binomial generating set of the toric ideal can be used to generate the associated Markov chain, Markov bases that make rapid connections between elements of all fibers should be preferred since our intuition tells us that these Markov chains will mix more rapidly. One desirable property of a Markov basis that guarantees short connections is the distance-reducing property [10]. Since Graver bases always satisfy the distance-reducing property, strongly robust toric ideals have the pleasing property that every Markov basis is distance-reducing. This suggests that strongly robust toric ideals should have nice properties from the standpoint of mixing times of the associated Markov chain.

Associated to the matrix $A$ is the Gale transform $B$ which is a $n \times(n-d)$ integer matrix whose columns span $\operatorname{ker}_{\mathbb{Z}} A$. When describing the matrix $A$, we often think about it as a list of column vectors $A=\left\{a_{1}, a_{2}, \ldots, a_{n}\right\}$. When describing the Gale transform we think about it as a list of row vectors $B=\left\{b_{1}, b_{2}, \ldots, b_{n}\right\}$. We assume throughout that the matrix $A$ has no co-loops, that is, $B$ does not contain the all zeros vector. With the no co-loop assumption, a bouquet is a maximal subset $S \subseteq[n] \operatorname{such}$ that $\operatorname{span}\left(b_{s}: s \in S\right)$ in one-dimensional. A bouquet $S$ is mixed if not all elements $\left\{b_{s}: s \in S\right\}$ lie in the same orthant. Note that our definition of bouquet differs from that of [7] but the two definitions are equivalent in the case that $A$ has no co-loops.

A key observation of [7] is that the toric ideals of Lawrence type have many mixed bouquets. Recall that if $A \in \mathbb{Z}^{d \times n}$, the Lawrence lifting of $A$ is the matrix

$$
\Lambda(A)=\left(\begin{array}{cc}
A & 0 \\
I & I
\end{array}\right) \in \mathbb{Z}^{(d+n) \times 2 n}
$$

where $I$ denotes an $n \times n$ identity matrix. A toric ideal $I_{C}$ is said to be of Lawrence type if it is equal to $I_{\Lambda(A)}$ for some matrix $A$, perhaps after permuting the indeterminates. Note that

$$
\operatorname{ker}_{\mathbb{Z}} \Lambda(A)=\left\{(u,-u) \in \mathbb{Z}^{2 n}: u \in \operatorname{ker}_{\mathbb{Z}} A\right\}
$$

A basis of $\operatorname{ker}_{\mathbb{Z}} \Lambda(A)$ must consist of vectors of the form $(u,-u)$. This will enforce that in the Gale transform $B, b_{i}=-b_{i+n}$ for all $i \in[n]$. When $A$ has no co-loops every $s \in[2 n]$ belongs to a mixed bouquet of $\Lambda(A)$, since $i$ and $n+i$ will be in the same bouquet. In fact, since rank of $B=\operatorname{codim} A$, we see that if a toric ideal is of Lawrence type it must have at least codim $A$ mixed bouquets. Petrović, Thoma, and Vladoiu also show how to use the bouquet structure to produce new examples of strongly robust toric ideals that are not of Lawrence type showing that the class of strongly robust toric ideals is not equal 
to the class of Lawrence ideals. They also posed the following question about strongly robust toric ideals.

Question 1.1. If $I_{A}$ is a strongly robust toric ideal, must $A$ have a mixed bouquet?

If $A \in \mathbb{Z}^{d \times n}$ is a toric ideal, with $d=\operatorname{rank} A$, then the codimension of $I_{A}$ is $n-d$. When the codimension of $I_{A}$ is one, in which case $I_{A}$ is a principal ideal, Question 1.1 is trivial since $A$ consists of a single bouquet that must be mixed if $I_{A}$ is positively graded. We also provide a positive answer to Question 1.1 in the case that $I_{A}$ has codimension 2 by giving a complete characterization of the strongly robust codimension 2 toric ideals in terms of the Gale transform, which is described in following sections. One consequence is the following:

Theorem 1.2. Let $A \in \mathbb{Z}^{(n-2) \times n}$ be a full rank matrix, and $\tilde{B}=\left\{\tilde{b}_{1}, \ldots, \tilde{b}_{n}\right\} \subseteq \mathbb{Z}^{2}$ be the reduced Gale transform of $A$. If $I_{A}$ is a strongly robust toric ideal then $\operatorname{conv}(\tilde{B})$ is a centrally symmetric polygon.

In fact, Theorem 1.2 provides a stronger answer to Question 1.1 in the case of codimension 2 toric ideals.

Corollary 1.3. If a codimension 2 toric ideal $I_{A}$ is strongly robust then $A$ has at least 2 mixed bouquets.

Both of these results will be a consequence of the general characterization of strongly robust codimension 2 toric ideals that we prove in the next section. The proof uses the Peeva-Sturmfels [6] theory of toric ideals of codimension 2. While the result of Theorem 1.2 does not directly generalize to toric ideals of higher codimension (see Example 2.9), it does suggest that the property of being strongly robust is connected to the geometry of the Gale transform, which might suggest other approaches to Question 1.1.

\section{Proof of Theorem 1.2 and Corollary 1.3}

To prove Theorem 1.2 we need more details about strongly robust toric ideals, and results about generating sets of codimension 2 toric ideals. Note that the main definitions and constructions will be illustrated in Example 2.8.

First of all, we need to formally introduce the definition of strongly robust toric ideal $[3,7]$. To explain this we introduce some definitions. Given a vector $u \in \mathbb{Z}^{n}$ the support of $u, \operatorname{supp}(u) \subseteq[n]$ is the set of indices $i$ where $u_{i}$ is not zero. Let $u \in \mathbb{N}^{n}$. The fiber of $u$ is the set $\mathcal{F}(u)=\left\{v \in \mathbb{N}^{n}: A u=A v\right\}$. Note that $p^{u}-p^{v} \in I_{A}$ if and only if $u, v$ belong to the same fiber. A binomial $p^{u}-p^{v}$ is called an indispensable binomial if $\mathcal{F}(u)=\{u, v\}$ and $\operatorname{supp}(u) \cap \operatorname{supp}(v)=\emptyset$. The set of all indispensable binomials is denoted by $\mathcal{S}(A)$. A binomial $p^{u}-p^{v} \in I_{A}$ is called primitive if there is no other binomial $p^{u^{\prime}}-p^{v^{\prime}} \in I_{A}$ such that $p^{u^{\prime}} \mid p^{u}$ and $p^{v^{\prime}} \mid p^{v}$. The set of all primitive binomials in $I_{A}$ is called the Graver basis of $A$, and denoted by $\mathcal{G} r(A)$. The universal Gröbner basis of $A$ is a subset of the Graver basis, and the set of indispensable binomials are a subset of the universal Gröbner basis. The set of indispensable binomials appear in every binomial minimal generating set of $I_{A}$. This leads to the following definition: 
Definition 2.1. The toric ideal $I_{A}$ is strongly robust if $\mathcal{S}(A)=\mathcal{G} r(A)$.

In [7] strongly robust toric ideals are called $\emptyset$-Lawrence. Every strongly robust toric ideal is robust. Boocher et al [3] wonder (Question 6.1) if robust implies strongly robust for toric ideals, and prove this is true in a family of toric ideals associated to graphs. At the time of this writing, the conjecture remains open.

One useful tool for analyzing the Graver bases of $I_{A}$, is its connection to the Lawrence lifting. Recall the definition of the Lawrence lifting $\Lambda(A)$ from the introduction. Its toric ideal $I_{\Lambda(A)}$ we consider to be in the ring $\mathbb{K}[p, q]$ with $2 n$ indeterminates. Binomials in $I_{\Lambda(A)}$ have the form $p^{u} q^{v}-p^{v} q^{u}$ such that $p^{u}-p^{v} \in I_{A}$.

Theorem 2.2. [8, Alg 7.2] Let $A \in \mathbb{Z}^{d \times n}$. Let $M=\left\{p^{u_{i}} q^{v_{i}}-p^{v_{i}} q^{u_{i}}: i=1, \ldots, m\right\}$ be $a$ binomial minimal generating set of the toric ideal $I_{\Lambda(A)}$. Then $\left\{p^{u_{i}}-p^{v_{i}}: i=1, \ldots, m\right\}$ is the Graver basis of $I_{A}$.

A key tool for studying toric ideals in codimension 2 are the reduced Gale diagrams. These were used by Peeva and Sturmfels [6] to give a complete description of the free resolution of codimension 2 toric ideals. We define them now:

Let $A \in \mathbb{Z}^{(n-2) \times n}$ be a matrix of rank $n-2$ and $B$ the resulting Gale configuration. Let $B=\left\{b_{1}, \ldots, b_{n}\right\}$ be the resulting list of row vectors, with $b_{i}=\left(b_{i 1}, b_{i 2}\right)$. The reduced Gale configuration $\tilde{B}=\left\{\tilde{b}_{1}, \ldots, \tilde{b}_{n}\right\}$ is obtained by setting

$$
\tilde{b}_{i}=\operatorname{gcd}\left(b_{i 1}, b_{i 2}\right)^{-1}\left(-b_{i 2}, b_{i 1}\right) .
$$

That is, $\tilde{B}$ is obtained from $B$ by rotating the vectors by 90 degrees and scaling so that elements in each vector are relatively prime. For the notion of a minimal generating set to be meaningful, we need to assume that the toric ideal $I_{A}$ is positively graded. In terms of the reduced Gale configuration, this means that there is no nonzero vector $w \in \mathbb{Q}^{2}$ such that $w^{T} \tilde{b}_{i}>0$ for all $i$. With this assumption, the vectors $\tilde{b}_{i}$ can be ordered in such a way that each pair $\tilde{b}_{i}, \tilde{b}_{i+1}$ span a cone such that no other $\tilde{b}_{j}$ lies in the interior of the cone (where $\left.\tilde{b}_{n+1}=\tilde{b}_{1}\right)$.

For each cone cone $\left(\tilde{b}_{i}, \tilde{b}_{i+1}\right)$, let $H_{i}$ be its Hilbert basis, which is the minimum generating set of the monoid cone $\left(\tilde{b}_{i}, \tilde{b}_{i+1}\right) \cap \mathbb{Z}^{2}$. Define the Hilbert basis of the reduced Gale configuration to be the set:

$$
\mathcal{H}_{A}=\left\{u \in \mathbb{Z}^{2} \text { : both } u \text { and }-u \text { are in } H_{1} \cup H_{2} \cup \cdots \cup H_{n}\right\} .
$$

Theorem 2.3. [6, Theorem 3.7] Let $A \in \mathbb{Z}^{(n-2) \times n}$ have rank $n-2$, and $B$ the Gale configuration. A vector $u \in \mathbb{Z}^{2}$ is in $\mathcal{H}_{A}$ if and only if $p^{(B u)_{+}}-p^{(B u)_{-}}$is an indispensable binomial of the toric ideal $I_{A}$. Furthermore, the indispensable binomials are a generating set for $I_{A}$, unless there are no indispensable binomials, in which case $I_{A}$ is a complete intersection.

Hilbert bases are complicated to compute for general cones, but in dimension 2 there is a particularly simple geometric description. 
Proposition 2.4. Let $a, b \in \mathbb{Z}^{2}$ and let $P=\operatorname{cone}(a, b)$. The Hilbert basis of $P$ consists of all lattice points in the polyhedron $\operatorname{conv}\left(\left(P \cap \mathbb{Z}^{2}\right) \backslash\{(0,0)\}\right)$ that are visible from the origin.

Proof. The set of lattice points in the polyhedron $\operatorname{conv}\left(\left(P \cap \mathbb{Z}^{2}\right) \backslash\{(0,0)\}\right)$ that are visible from the origin can be ordered as $b_{1}, b_{2}, \ldots, b_{k}$ (say in clockwise orientation as viewed from the origin). This induces a triangulation of $P$ into cones cone $\left(b_{i}, b_{i+1}\right)$. Since, by assumption, the triangle $\operatorname{conv}\left(\left\{(0,0), b_{i}, b_{i+1}\right\}\right)$ only contains those three lattice points, this is a unimodular triangle, and so the cone cone $\left(b_{i}, b_{i+1}\right)$ is unimodular. The unimodularity of cone $\left(b_{i}, b_{i+1}\right)$ means that every lattice point in cone $\left(b_{i}, b_{i+1}\right)$ can be written as a nonnegative integer combination of $b_{i}$ and $b_{i+1}$. This shows that all lattice points in $P$ can be written as nonnegative integer combinations of $b_{1}, b_{2}, \ldots, b_{k}$. Finally, none of $b_{1}, b_{2}, \ldots, b_{k}$ can be omitted because that lattice point cannot be written as a nonnegative integer combination of the others. This shows that $b_{1}, b_{2}, \ldots, b_{k}$ is a Hilbert basis as desired.

Combining Theorems 2.2 and 2.3, the Graver basis of $A$ can also be characterized in terms of the reduced Gale configuration.

Corollary 2.5. Let $A \in \mathbb{Z}^{(n-2) \times n}$ have rank $n-2$, and $B$ the Gale configuration. Suppose that $\operatorname{ker}_{\mathbb{Z}} A \cap \mathbb{N}^{n}=\{0\}$. A vector $u \in \mathbb{Z}^{2}$ has either $u$ or $-u \in H_{1} \cup \cdots \cup H_{n}$ if and only if $p^{(B u)_{+}}-p^{(B u)_{-}}$is a primitive binomial of the toric ideal $I_{A}$.

Proof. For the Gale configuration $B$ of $A$, define $B^{ \pm}:=B \cup-B$, which is the Gale configuration of the Lawrence lifting $\Lambda(A)$, and let $\tilde{B}^{ \pm}$be its reduced Gale configuration. As for $\tilde{B}$, we assume that the elements of $\tilde{B}^{ \pm}$are ordered so that for each cone cone $\left(\tilde{b}_{i}, \tilde{b}_{i+1}\right)$ no other $\tilde{b}_{j}$ lies in its interior. Let $H_{i}^{ \pm}$be the Hilbert basis of cone $\left(\tilde{b}_{i}, \tilde{b}_{i+1}\right)$. Note that $\tilde{B}^{ \pm}$is centrally symmetric. This means that if $u$ is in the Hilbert basis of some $H_{i}^{ \pm}$then $-u$ is in the Hilbert basis of $-H_{i}^{ \pm}$. Thus the Hilbert basis of the resulting Lawrence configuration $\Lambda(A)$ is the union of all the $H_{i}^{ \pm}$. By Theorem 2.3 these vectors determine the minimal generating set of $I_{\Lambda(A)}$. By Theorem 2.2 those vectors then determine the Graver basis of $I_{A}$. So to prove the corollary, we need to show that for every $u$ in some $H_{i}^{ \pm}$, either $u$ or $-u$ appears in $H_{1} \cup \cdots \cup H_{n}$.

So let $u \in H_{i}^{ \pm}$. If $\tilde{b}_{i}$ and $\tilde{b}_{i+1}$ are both in $\tilde{B}$ or both in $-\tilde{B}$, then cone $\left(\tilde{b}_{i}, \tilde{b}_{i+1}\right)$ or cone $\left(-\tilde{b}_{i},-\tilde{b}_{i+1}\right)$ is one of the cones described in the Hilbert basis of $A$, so $u$ or $-u$ belongs to $H_{1} \cup \cdots \cup H_{n}$. This leaves the case that $\tilde{b}_{i} \in \tilde{B}$ and $\tilde{b}_{i+1} \in-\tilde{B}$ (the reverse situation follows from a symmetric argument). Looking at the ordering on $\tilde{B}$, there will be a unique smallest $j$ such that $\tilde{b}_{j} \in \tilde{B}$ and cone $\left(\tilde{b}_{i}, \tilde{b}_{j}\right)$ forms one of the cones for computing $H_{1} \cup \cdots \cup H_{n}$. Similarly, there is a unique largest $k$ such that $-\tilde{b}_{k} \in \tilde{B}$ and cone $\left(-\tilde{b}_{k},-\tilde{b}_{i+1}\right)$ forms one of the cones for computing $H_{1} \cup \cdots \cup H_{n}$. These vectors are guaranteed to exist by the positive grading assumption that $\operatorname{ker}_{\mathbb{Z}} A \cap \mathbb{N}^{n}=\{0\}$. Note that by the way that we have chosen $\tilde{b}_{j}$ and $\tilde{b}_{k}$ we have

$$
\operatorname{cone}\left(\tilde{b}_{i}, \tilde{b}_{i+1}\right)=\operatorname{cone}\left(\tilde{b}_{i}, \tilde{b}_{j}\right) \cap \operatorname{cone}\left(\tilde{b}_{k}, \tilde{b}_{i+1}\right) .
$$

Furthermore, if we let

$$
P_{i, i+1}=\operatorname{conv}\left(\operatorname{cone}\left(\tilde{b}_{i}, \tilde{b}_{i+1}\right) \cap \mathbb{Z}^{2} \backslash\{(0,0)\}\right)
$$


and defined $P_{i, j}$ and $P_{k, i+1}$ similarly, then we have that

$$
P_{i, i+1}=P_{i, j} \cap P_{k, i+1} .
$$

Since each of $P_{i, i+1}, P_{i, j}$, and $P_{k, i+1}$ is the convex hull of lattice points, the lattice points visible from the origin in $P_{i, i+1}$, will be either a lattice point visible from the origin in $P_{i, j}$ or in $P_{k, i+1}$, or both. In the case that $u \in P_{i, i+1}$ is a lattice point visible from the origin with $u \in P_{i, j}$, then $u \in H_{1} \cup \cdots \cup H_{n}$. In the case that $u \in P_{i, i+1}$ is a lattice point visible from the origin with $u \in P_{k, i+1}$ then $-u \in H_{1} \cup \cdots \cup H_{n}$.

Corollary 2.5 then reduces the problem of characterizing strongly robust toric ideals in codimension 2 to the following problem.

Problem 2.6. For which rank $n-2$ matrices $A \in \mathbb{Z}^{(n-2) \times n}$ is the Hilbert basis $\mathcal{H}_{A}$ equal to $H_{1} \cup H_{2} \cup \cdots \cup H_{n}$ ?

The answer is contained in the following lemma.

Lemma 2.7. Let $A \in \mathbb{Z}^{(n-2) \times n}$ have rank $n-2$ and $\tilde{B}$ the reduced Gale diagram. Then $I_{A}$ is strongly robust if and only if for each $\tilde{b}_{i} \in \tilde{B},-\tilde{b}_{i} \in \mathcal{H}_{A}$.

Proof. $(\Longrightarrow)$ Suppose that $I_{A}$ is strongly robust. Then $\mathcal{H}_{A}=H_{1} \cup \cdots \cup H_{n}$. Since $\tilde{b}_{i} \in H_{i}$, the definition of $\mathcal{H}_{A}$ forces that $-\tilde{b}_{i} \in \mathcal{H}_{A}$.

$(\Longleftarrow)$. Conversely, suppose that for each $\tilde{b}_{i} \in \tilde{B}$ we have $-\tilde{b}_{i} \in \mathcal{H}_{A}$. We will show that $\mathcal{H}_{A}=H_{1} \cup \cdots \cup H_{n}$. Now if $-\tilde{b}_{i} \in \mathcal{H}_{A}$ but $-\tilde{b}_{i} \notin \tilde{B}$, we can add it to $\tilde{B}$ without changing $\mathcal{H}_{A}$ or $H_{1} \cup \cdots \cup H_{n}$ although there is one more new cone. Indeed, if cone $\left(\tilde{b}_{j}, \tilde{b}_{j+1}\right)$ contains $-\tilde{b}_{i}$ as a visible lattice point of $P_{j, j+1}$, then the visible lattice points arising in the cones cone $\left(\tilde{b}_{j},-\tilde{b}_{i}\right)$ and cone $\left(-\tilde{b}_{i}, \tilde{b}_{j+1}\right)$ are precisely the visible lattice points in $P_{j, j+1}$. By repeating this procedure, we end up with a configuration of vectors that contains only pairs $\tilde{b}_{i},-\tilde{b}_{i}$, which is the reduced Gale diagram of a Lawrence matrix. Such a configuration automatically satisfies the condition that $\mathcal{H}_{A}=H_{1} \cup \cdots \cup H_{n}$ by Theorem 2.3 and Corollary 2.5. However, since our operations did not change either the sets $\mathcal{H}_{A}$ or $H_{1} \cup \cdots \cup H_{n}$, we see that $I_{A}$ is strongly robust.

Now we are in a position to prove Theorem 1.2.

Proof. [Proof of Theorem 1.2] Suppose that $I_{A}$ is a strongly robust toric ideal in codimension 2 , let $\tilde{B}$ be the reduced Gale configuration, and $P=\operatorname{conv}(\tilde{B})$ the convex hull of the elements in $\tilde{B}$. Let $\tilde{b}$ be a vertex of $P$. We must show that $-\tilde{b}$ is also a vertex of $P$ to see that $P$ is centrally symmetric.

Since $\tilde{b} \in B$, and $I_{A}$ is strongly robust, $-\tilde{b}$ belongs to $\mathcal{H}_{A}$, by Lemma 2.7. If $-\tilde{b}$ is not a vertex of $P$, then there are two vectors $\tilde{b}_{1}, \tilde{b}_{2} \in \tilde{B}$ such that $-\tilde{b}$ is in $\operatorname{conv}\left(\tilde{b}_{1}, \tilde{b}_{2},(0,0)\right)$. Applying Lemma 2.7 again, we have the $-\tilde{b}_{1}$ and $-\tilde{b}_{2}$ are in $\mathcal{H}_{A}$. In particular, these two vectors are in $P$, by Proposition 2.4. However, this forces that $\tilde{b} \in \operatorname{conv}\left(-\tilde{b}_{1},-\tilde{b}_{2},(0,0)\right)$, so $\tilde{b}$ could not be a vertex of $P$.

Proof. [Proof of Corollary 1.3] Since the polytope $\operatorname{conv}(\tilde{B})$ must be two dimensional and is centrally symmetric, it must have at least two pairs of opposite vertices $\tilde{b}_{1},-\tilde{b}_{1}$ and $\tilde{b}_{2},-\tilde{b}_{2}$. These two pairs of opposite vertices yield two mixed bouquets of the matrix $A$. 


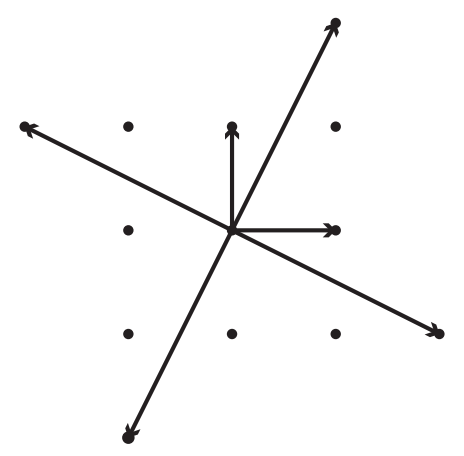

Figure 1: The reduced Gale transform of the matrix in Example 2.8. The dots represent the points in the set $\mathcal{H}_{A}$.

Example 2.8. Let $A$ be the matrix

$$
A=\left(\begin{array}{cccccc}
1 & 0 & 1 & 0 & 0 & 0 \\
0 & 1 & 0 & 0 & 1 & 0 \\
0 & 1 & 0 & 1 & 0 & 1 \\
-2 & 0 & 0 & 0 & -4 & 5
\end{array}\right)
$$

which has the Gale transform $B$ and reduced Gale transform $\tilde{B}$ respectively:

$$
B=\left(\begin{array}{cc}
1 & 2 \\
-2 & 1 \\
-1 & -2 \\
0 & -1 \\
2 & -1 \\
2 & 0
\end{array}\right) \quad \tilde{B}=\left(\begin{array}{cc}
-2 & 1 \\
-1 & -2 \\
2 & -1 \\
1 & 0 \\
1 & 2 \\
0 & 1
\end{array}\right)
$$

As can be seen from the reduced Gale transform, illustrated in Figure 1, the matrix $A$ satisfies the condition of Lemma 2.7, and so the toric ideal is strongly robust. The minimal generating set, which equals the Graver basis, consists of the following 6 binomials that are in bijection with pairs of opposite lattice points in $\mathcal{H}_{A}$ :

$$
I_{A}=\left\langle b^{5}-d e^{5} f^{4}, a e^{2} f^{2}-b^{2} c, a b^{3}-c d e^{3} f^{2}, a^{2} b-c^{2} d e, a^{3} e f^{2}-b c^{3} d, a^{5} f^{2}-c^{5} d^{2}\right\rangle .
$$

For example, the binomial $a^{3} e f^{2}-b c^{3} d$ corresponds to the point $u=(1,1)$ in the Gale diagram, since $B u=(3,-1,-3,-1,1,2)^{T}$.

Finally, we conclude with an example of a strongly robust toric ideal of codimension 5 such that the convex hull of the reduced Gale transform is not centrally symmetric. This shows that the direct generalization of Theorem 1.2 is not true for higher codimension.

Example 2.9. Consider the following matrix $A$ and its reduced Gale transform $\tilde{B}$ (where we divide by the greatest common divisor of each row entry), which comes from Example $4.3(\mathrm{~b})$ of [7]. 


$$
A=\left(\begin{array}{ccccccccccccccc}
1 & 1 & 1 & 0 & 0 & 0 & 0 & 0 & 0 & 0 & 0 & 0 & 0 & 0 & 0 \\
1 & 0 & 0 & 1 & 0 & 0 & 0 & 0 & 0 & 0 & 0 & 0 & 0 & 0 & 0 \\
0 & 1 & 0 & 0 & 1 & 0 & 0 & 0 & 0 & 0 & 0 & 0 & 0 & 0 & 0 \\
0 & 0 & 0 & 0 & 0 & 1 & 1 & 1 & 0 & 0 & 0 & 0 & 0 & 0 & 0 \\
0 & 0 & 0 & 0 & 0 & 1 & 0 & 0 & 1 & 0 & 0 & 0 & 0 & 0 & 0 \\
0 & 0 & 0 & 0 & 0 & 0 & 1 & 0 & 0 & 1 & 0 & 0 & 0 & 0 & 0 \\
0 & 0 & 0 & 0 & 0 & 0 & 0 & 0 & 0 & 0 & 1 & 1 & 1 & 0 & 0 \\
0 & 0 & 0 & 0 & 0 & 0 & 0 & 0 & 0 & 0 & 1 & 0 & 0 & 1 & 0 \\
0 & 0 & 0 & 0 & 0 & 0 & 0 & 0 & 0 & 0 & 0 & 1 & 0 & 0 & 1 \\
0 & 0 & 1 & 1 & 1 & 0 & 0 & 1 & 1 & 1 & 0 & 0 & 1 & 1 & 1
\end{array}\right) \quad \tilde{B}=\left(\begin{array}{ccccc}
0 & 1 & 0 & 0 & 0 \\
0 & -1 & 1 & -1 & 0 \\
0 & 0 & -1 & 1 & 0 \\
0 & -1 & 0 & 0 & 0 \\
0 & 1 & -1 & 1 & 0 \\
1 & 0 & 0 & 1 & 0 \\
-1 & -1 & 1 & 0 & 1 \\
0 & 1 & -1 & -1 & -1 \\
-1 & 0 & 0 & -1 & 0 \\
1 & 1 & -1 & 0 & -1 \\
0 & 1 & -2 & -1 & -2 \\
0 & 0 & 0 & 1 & 1 \\
0 & -1 & 2 & 0 & 1 \\
0 & -1 & 2 & 1 & 2 \\
0 & 0 & 0 & -1 & -1
\end{array}\right)
$$

The fact that this yields a strongly robust toric ideal was verified in [7]. This example has 6 mixed bouquets, as can be seen from inspecting $\tilde{B}$. On the other hand, the convex hull of the set of rows of $\tilde{B}$ has 15 vertices. Since centrally symmetric polytopes always have an even number of vertices, this polytope cannot be centrally symmetric.

On the other hand, we know of no counterexample to the natural generalization of Corollary 1.3, namely: Does every strongly robust toric ideal of codimension $r$ have $r$ mixed bouquets?

\section{Acknowledgments}

Seth Sullivant was partially supported by the David and Lucille Packard Foundation and the US National Science Foundation (DMS 1615660).

\section{References}

[1] Federico Ardila and Adam Boocher. The closure of a linear space in a product of lines. J. Algebraic Combin., 43(1):199-235, 2016.

[2] David Bernstein and Andrei Zelevinsky. Combinatorics of maximal minors. J. Algebraic Combin., 2(2):111-121, 1993.

[3] Adam Boocher, Bryan Christopher Brown, Timothy Duff, Laura Lyman, Takumi Murayama, Amy Nesky, and Karl Schaefer. Robust graph ideals. Ann. Comb., 19(4):641-660, 2015.

[4] Adam Boocher and Elina Robeva. Robust toric ideals. J. Symbolic Comput., 68(1):254-264, 2015.

[5] Persi Diaconis and Bernd Sturmfels. Algebraic algorithms for sampling from conditional distributions. Ann. Statist., 26(1):363-397, 1998. 
[6] Irena Peeva and Bernd Sturmfels. Syzygies of codimension 2 lattice ideals. Math. Z., 229(1):163-194, 1998.

[7] Sonja Petrović, Apostolos Thoma, and Marius Vladoiu. Bouquet algebra of toric ideals. J. Algebra, 512:493-525, 2018.

[8] Bernd Sturmfels. Gröbner bases and convex polytopes, volume 8 of University Lecture Series. American Mathematical Society, Providence, RI, 1996.

[9] Bernd Sturmfels and Andrei Zelevinsky. Maximal minors and their leading terms. Adv. Math., 98(1):65-112, 1993.

[10] Akimichi Takemura and Satoshi Aoki. Distance-reducing Markov bases for sampling from a discrete sample space. Bernoulli, 11(5):793-813, 2005. 